\title{
Oral Steroids in Initial Treatment of Acute Sciatica
}

\author{
Richard L. Holve, MD, and Howard Barkan, DrPH
}

Objective: Many physicians use prednisone to treat acute sciatica with the hope of speeding recovery. There is little clinical evidence to support this practice. Our objective was to determine whether early administration of oral prednisone affects parameters related to recovery from acute sciatica.

Methods: In this double-blind, controlled clinical trial, 27 patients were sequentially assigned to receive either a 9-day tapering course of prednisone $(n=13)$ or placebo $(n=14)$ within 1 week of developing sciatic symptoms. Patients and investigators were blinded to the drug administered. Follow-up assessment was done weekly for 1 month and then monthly for 5 months.

Results: Prednisone and control groups showed no statistically significant differences in physical findings, use of nonsteroidal anti-inflammatory drugs or narcotic medications, or rates of patients returning to work at any time interval studied. Compared with controls, patients who received prednisone had more rapid rates of improvement from baseline in pain, mental well-being, and disability scores. These changes were subtle but statistically significant. Patients who received prednisone tended to receive fewer epidural injections for pain.

Conclusions: Early administration of oral steroid medication in patients with acute sciatica had no significant effect on most parameters studied. It did, however, lead to slightly more rapid rates of improvement in pain, mental well-being, and disability scores. The impact of oral steroids on other outcomes is suggested by this study, but its small sample size limited its statistical power. (J Am Board Fam Med 2008;21:469-474.)

Sciatica (lumbosacral radiculopathy) is a common problem seen by primary care physicians and is diagnosed in approximately $1 \%$ of all patients who present with acute low back pain. ${ }^{1,2}$ Traditional treatment includes pain medication (acetaminophen, nonsteroidal anti-inflammatory agents [NSAIDs], or narcotic agents); activity as tolerated; and time. ${ }^{1,3-8}$ Patients with intractable pain or progressive neurologic symptoms usually receive an epidural injection of steroid drugs and, if necessary, decompressive laminectomy or diskectomy. ${ }^{2,8,9}$ Fifty percent of patients with sciatica recover within 6 weeks and $90 \%$ recover within 12 weeks. ${ }^{4,8}$ Despite being a mostly self-limiting condition, sciatica results in tremendous loss to our society in terms of de-

This article was externally peer reviewed.

Submitted 25 September 2007; revised 28 February 2008; accepted 3 March 2008.

From the Department of Family Medicine, Kaiser Permanente Medical Center, Santa Rosa; and Department of Surgery, Kaiser Permanente Medical Center, Oakland, CA. Funding: This trial was funded by the Kaiser Foundation Research Institute.

Conflict of interest: none declared.

Corresponding author: Richard Holve, MD, Department of Family Practice, Kaiser Permanente Medical Center, Santa Rosa, CA 95403 (E-mail: Rich.Holve@kp.org). creased productivity, disability, and treatment costs. These costs are incurred both during the initial episode and over the long term. ${ }^{1,10}$

Sciatica results from mechanical and/or inflammatory events that affect one or more lumbosacral nerve roots. ${ }^{11}$ Nerve root compression by a herniated disk is thought to be the most common cause of sciatica. ${ }^{12}$ Tissue damage results in an autoimmune response that produces proinflammatory substances that affect the neuraxial system. ${ }^{13}$ Studies of patients who have sustained injury to the spinal cord or nerve roots suggest that pain and disability result from a complex interplay between local inflammatory responses and neurohumeral interaction between the brain, spinal cord, and nerve roots. ${ }^{13-15}$

Steroid agents are known to reduce tissue damage by stabilizing cell membranes, reducing capillary permeability, and limiting release of proinflammatory substances. ${ }^{13}$ Based on this knowledge, it has become relatively common for primary care physicians to treat acute sciatica with a course of oral steroids, although there is little clinical evidence to support this practice. ${ }^{6,15}$ Literature searches using PUBMED and MEDLINE pro- 
duced only one blinded, randomized, controlled study that addressed this issue. ${ }^{15}$ In that study, performed in the 1980s, all patients were hospitalized and put on bed rest for 1 week. The study group received a 7-day tapering dose of oral dexamethasone, which was observed to be no better than placebo for treating sciatic pain. ${ }^{15}$ Other studies have shown mixed, but generally positive, shortterm results from treatment of sciatica using epidural injection of steroid agents. ${ }^{2,6,8,12,16-21}$

Contemporary treatment of acute sciatica occurs almost exclusively in the outpatient arena. We sought to determine whether the use of oral steroids truly reduces pain and disability in this setting. We hypothesized that the use of prednisone as part of the initial treatment for acute sciatica would speed patients' recovery as measured by changes in physical findings, rates of return to work, ability to conduct activities of daily living, scores assessing levels of pain and disability, use of narcotic drugs and NSAIDs, and need for epidural injection or surgical intervention. We conducted a doubleblind clinical trial to assess this hypothesis.

\section{Methods}

Patients were recruited from among those seen for new onset back pain in the adult primary care or emergency departments at the Kaiser Permanente Medical Center in Santa Rosa, California. The study was conducted with the full approval of the Kaiser Permanente Northern California Institutional Review Board. Each study subject signed an informed consent form that met guidelines set forth by the United States Department of Health and Human Services Office for Human Research Protections.

All patients were assessed by the principal investigator $(\mathrm{RLH})$ on referral to the study to ensure that they met all 3 study eligibility criteria: a diagnosis of acute sciatica, age between 20 and 60 years, and recruitment into the study within 1 week after onset of symptoms. The principal investigator confirmed the diagnosis based on finding unilateral leg pain extending below the knee and a positive straight-leg-raising sign (defined as pain radiating from the buttock to below the knee with elevation of the leg between $0^{\circ}$ and $60^{\circ}$ ).

Patients were excluded from the study if they were pregnant or had a history of diabetes, renal failure, upper gastrointestinal bleeding, or major psychiatric disease. Exclusion criteria also included the presence of symptoms and/or a medical history that suggested increased risk for serious underlying disease as defined by the United States Agency for Health care Policy Research document, "Acute Low Back Problems in Adults." "Red flag" symptoms suggesting such disease include a history of cancer; unexplained weight loss; fever, chills, or night sweats; a history of intravenous drug use; saddle anesthesia; bowel or bladder incontinence; bone pathology; or a neurologic emergency. ${ }^{3} \mathrm{~Pa}$ tients could also be excluded if they had any condition which the principal investigator believed might jeopardize their safety.

\section{Treatment Assignment and Blinding}

Once the diagnosis of acute sciatica had been confirmed, the principle investigator or the research nurse obtained informed consent from all patients and referred them to a pharmacist. The pharmacy department used its standard protocol to assign these patients to receive either a 9-day tapering course of prednisone or placebo capsules. According to this protocol, subjects were sequentially entered into the study; odd-numbered patients received prednisone and even-numbered patients received placebo. The principle investigator and research nurse were blinded as to the specific group assignment and to the methods used to make that assignment.

\section{Study Design}

All patients received current standard therapy for sciatica including: a NSAID (ibuprofen, naproxen, etodolac, or nabumetone); narcotics for pain control, if needed (hydrocodone, propoxyphene, oxycodone, or morphine); activity as tolerated; and a referral for physical therapy. Patients randomized to the study group received a tapering course of prednisone: $60 \mathrm{mg}$ for 3 days, $40 \mathrm{mg}$ for 3 days, and $20 \mathrm{mg}$ for 3 days. Patients randomized to the control group received capsules identical in appearance to the prednisone capsules but containing an inert filler substance. At each visit during the first 9 days of the study, patients were questioned about their adherence to the prescribed course of study medication or placebo. Throughout the study, patients were questioned about whether they were still taking NSAIDs or narcotic medication and about the presence and magnitude of leg and back pain. 
During the initial visit and all subsequent visits, specific attention was given to 8 measurement parameters: ipsilateral straight-leg-raising sign; contralateral straight-leg-raising sign; knee and ankle stretch reflexes; foot sensation; strength of quadriceps, foot dorsiflexors, and foot plantarflexors; and ability to perform 5 heel lifts. At each visit, patients completed 3 written questionnaire instruments: the 12-item Health Status Questionnaire (SF-12), ${ }^{22}$ the Roland-Morris Disability Questionnaire, ${ }^{23}$ and the Roland-Morris Pain Rating Scale. ${ }^{23}$ Also noted during each visit were the number of hours the patient was employed or, if the patient was not working, the estimated percentage of daily living activities which the patient had been able to accomplish in the interval since the previous visit. Note was made of whether the patient had received an epidural injection of steroid medication or a surgical intervention since the previous visit. Each patient received the same examination and completed the same questionnaires at intake and weekly for 4 weeks, then monthly for 5 months. Total follow-up time was thus 6 months.

Patients were encouraged to begin non-weightbearing aerobic activity such as swimming and bicycle riding as soon as their pain had subsided to a reasonable degree. Most patients were also offered referral to a physical therapist.

Imaging studies were not done for patients who were younger than 50 years of age and who had rapid improvement of symptoms. Plain radiograph films and magnetic resonance imaging (MRI) scans of the lumbosacral spine were obtained for patients who had intractable pain or progressive neurologic symptoms.

\section{Statistical Analysis}

We designed the study to use standard inferential statistics for independent samples to compare results in prednisone and control groups. We used Fisher's exact test, $\chi^{2}$ analyses, Mann-Whitney U test, and Student's $t$ test to compare between the prednisone and control groups at each measurement time. The Wilcoxon signed-rank test was used to make intragroup comparisons of measures obtained at specified intervals from baseline. We designed the study to have adequate statistical power (power, $80 \%$ at $P<.05$ ) to test the hypothesis that prednisone given within the first week after appearance of symptoms is effective in $25 \%$ of patients for whom conservative treatment failed
(nQuery Advisor, version 5.0, 2002, Statistical Solutions, Sagas, MA). Results in table format are readily available from the authors on request.

\section{Results}

\section{Characteristics of the Study Sample}

Twenty-nine patients presenting to our medical center within 1 week of developing sciatic symptoms were recruited into this study. Fourteen patients were randomized to the control group and 15 to the prednisone group. Because of time conflicts, 2 patients in the prednisone group quit during the first week, leaving 14 control and 13 prednisone treated subjects. Seventeen subjects $(63.0 \%$; $95 \%$ CI, 42.4-80.6) were men and 10 (37.0\%; 95\% CI, 19.4-57.6) were women. The association between gender and randomization assignment was not statistically significant (Fisher's exact test; $P=.70$, not significant). Sex of the patient did not have a statistically significant association with any of the principal outcome measures. Overall mean age at intake was 42.59 years ( $95 \%$ CI, 38.38-46.81). For the control group, mean age at intake was 45.64 years (95\% CI, 39.78-51.51) whereas the average age at intake of prednisone group members was 39.31 years (95\% CI, 32.92-45.70). The difference in average age between the members of the study group and the members of the control group was not statistically significant (Student's $t$ test, 1.59; $P=.13$, not significant). Neither sex nor age was associated with statistically significant differences in baseline outcome measures.

\section{Physical Examination Measures}

No clinically significant differences in strength, sensation, reflexes, or straight leg raising tests were observed between control and prednisone groups at any specific time during this study.

\section{Comparison of Pain Scores}

Wilcoxon signed-rank tests found that pain scores in the prednisone group had statistically significant improvement from baseline at all durations of follow-up beginning at 1 week post treatment. In contrast, with the exception of week 2, differences from baseline within the control group did not become statistically significant until week 4 . Between month 2 and month 6, both groups had similar rates and degrees of improvement in pain scores (Figure 1). Pain scores did not differ signif- 


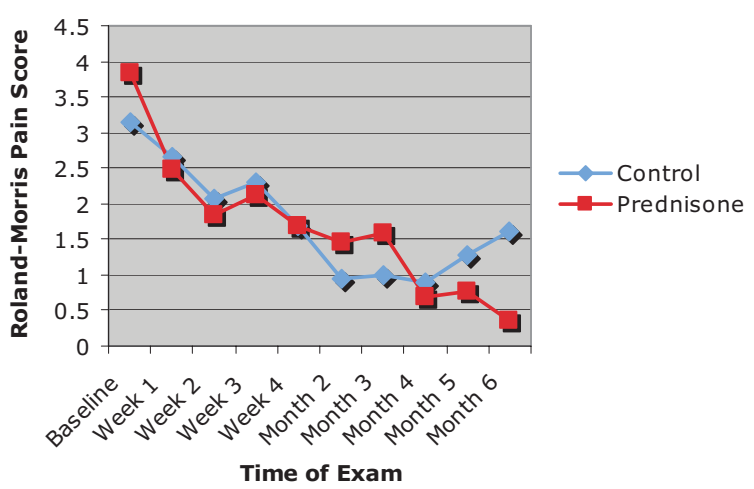

Figure 1. Roland-Morris pain scores. Intragroup changes measured over specified intervals from baseline.

icantly between control and prednisone groups at any specific time during the study.

\section{Comparison of Mental And Physical Health Scores}

Wilcoxon signed-rank tests found the prednisone group to have statistically significant improvement from baseline by week 4, whereas the change in mental health scores for the control group became statistically significant at a later time (Figure 2). Scores for well being (mental and physical) did not differ significantly between control and prednisone groups at any specific time during the study.

\section{Disability Comparison}

Wilcoxon signed-rank tests found that patients in the prednisone group had statistically significant improvement from baseline by weeks 2 and 3, whereas differences from baseline for the control group did not become statistically significant until

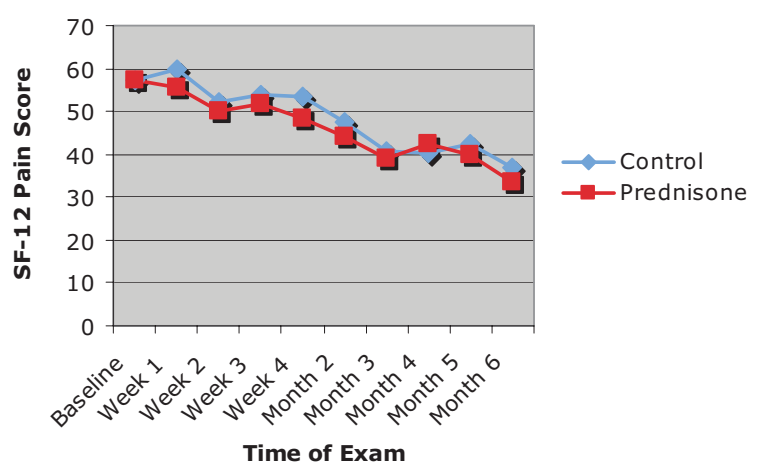

Figure 2. SF-12 mental health scores. Intragroup changes measured over specified intervals from baseline.

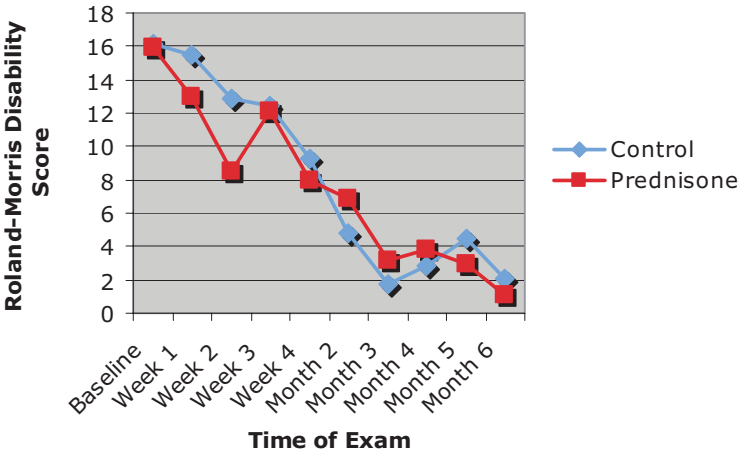

Figure 3. Roland-Morris disability scores. Intragroup changes measured over specified intervals from baseline.

the fourth week of the study (Figure 3). Both groups showed statistically significant improvement at week 4 through month 6 of the study. No statistically significant differences in disability scores were observed between the control and prednisone groups at any specific time in the study.

\section{Return To Work Comparison}

As expected, both the prednisone and control groups worked fewer hours during the first 4 weeks after the onset of sciatic symptoms. By the second month of the study, approximately $60 \%$ of subjects in each group had returned to their baseline work hours. No statistically significant differences were observed between the number of control or prednisone patients engaged in full- or part-time work or other activities during any specific point or interval of the study.

\section{Medication Use}

No statistically significant differences in use of narcotic drugs or NSAIDs were observed between control and prednisone groups at any time or interval during the study. No clinically significant adverse events related to medication were reported by patients in either group.

\section{Imaging Studies}

Plain radiograph films of the lumbosacral spine were obtained for 2 patients in the control group and for 4 patients in the prednisone group. For 7 patients in each group, MRI scanning was done based on progressive neurologic symptoms or intractable pain. All patients who underwent MRI 
had clinically significant disk and nerve root abnormalities.

\section{Epidural Injection and Surgical Intervention}

Epidural injection was administered to 2 of 13 patients $(15.4 \%)$ in the prednisone group compared with 6 of 14 patients (42.9\%) in the control group. The trend in this comparison is clear, although sample-size limitations prevented these results from reaching statistical significance. One patient in the control group ultimately underwent an L5 diskectomy.

\section{Discussion}

In our study, patients with acute sciatica who received prednisone within the first week of developing symptoms showed no significant differences from control patients at any follow-up interval in terms of physical findings, use of narcotic drugs or NSAIDs, or rates at which patients returned to work. Patients who received prednisone did have slightly more rapid rates of improvement in pain, mental well-being, and disability scores that were statistically significant. Patients who received prednisone also required fewer epidural injections than did members of the control group.

Our study confirms, in a contemporary outpatient setting, as Haimovic's study ${ }^{15}$ did with hospitalized patients 20 years ago, that there are no dramatic results to be gained from treating acute sciatica with oral steroids. However, patients in our study who received prednisone experienced statistically significant, although subtle, improvements that should not be discounted. These results are consistent with the findings of studies ${ }^{17-19}$ in which epidural injection produced the greatest benefit within 2 to 6 weeks after treatment. Such effects make sense in light of 2 factors: (1) the physiologic changes that occur with nerve root injury (release of pro-inflammatory substances) and (2) the pharmacokinetics of steroid medication (stabilization of cell membranes). ${ }^{13,14}$ An alternative explanation for our findings might be the influence of prednisone on mood apart from it's anti-inflammatory effect. A small percentage of patients receiving prednisone may experience anxiety, depression, mood elevation, or insomnia. ${ }^{24}$ Previous studies on the effects of prednisone on multiple parameters in the treatment of chronic obstructive lung disease and asthma have demonstrated conflicting effects on mood. ${ }^{25,26}$ A recent randomized crossover study looking specifically at the effect of prednisone on mood showed no significant differences between patients who received $60 \mathrm{mg}$ of prednisone for 3 days and those who received placebo. ${ }^{27}$

Physicians who choose to use prednisone in the initial treatment of acute sciatica should be sure they select patients with clear-cut signs and symptoms of this condition-not every patient with a complaint of back and leg pain has lumbosacral radiculopathy. They should discuss the risks and benefits of prednisone with patients, and realize that the benefits of this treatment are likely to be modest in the short term based on current clinical evidence.

The strength of our conclusions is limited by our study's relatively small sample size. These sample-size limitations were the result of simple logistic difficulties. The use of a single clinical investigator enhanced consistency, but the labor-intensive nature of each visit limited the number of patients who could be seen during any given period. We have collected a great deal of data on multiple parameters that may allow future researchers to focus their attention on more selective outcomes in a larger group of patients. An intriguing question raised by our study is whether oral steroids might reduce the need for epidural injection or surgery and, by implication, limit work disability and overall health costs related to this condition in the long term. We recommend that a larger, randomized, blinded study be done examining patients with acute sciatica who receive prednisone versus placebo, with focus on 2 "hard" endpoints: (1) return to work and (2) the number of epidural injections and surgeries performed.

Susan Kirk, RN, CCRC; Terry Hess, MA; and Danielle Rivers, CMA, assisted in data collection and organization. Editorial assistance was provided by the Medical Editing Service of The Permanente Medical Group Physician Education and Development Department.

\section{References}

1. Frymoyer JW. Back pain and sciatica. N Eng J Med 1988;318:291-300.

2. Scheer SJ, Radack KL, O'Brien DR Jr. Randomized controlled trials in industrial low back pain relating to return to work. Part 2. Discogenic low back pain. Arch Phys Med Rehab 1996;77:1189-97.

3. Bigos S, Bowyer O, Braen G, et al. Acute Low Back Problems in Adults. Clinical Practice Guide- 
line No. 14. AHCPR Publication No. 95-0642. Rockville, MD: Agency for Health Care Policy and Research, Public Health Service, U.S. Department of Health and Human Services. December 1994. Available from: http://www.ncbi.nlm.nih. gov/books/bv.fcgi? rid=hstat6.chapter.25870. Accessed 11 July 2008.

4. Daniels JM. Treatment of occupationally acquired low back pain. Am Fam Phy 1997;55:587-96.

5. Deyo RA. Conservative therapy for low back pain: distinguishing useful from useless therapy. JAMA 1983;250:1057-62.

6. Deyo RA. Back pain revisited. Newer thinking on diagnosis and therapy. Consultant 1993;88-97.

7. Griffin G, Tudiver F, Grant WD. Do NSAIDs help in acute or chronic low back pain? Am Fam Phy 2002;65:1319-21.

8. Weber H. The natural history of disc herniation and the influence of intervention. Spine 1994;19:2234-8; discussion, 2333.

9. Kraemer J. Presidential address: natural course and prognosis of intervertebral disc diseases. International Society for the Study of the Lumbar Spine, Seattle, Washington, June 1994. Spine 1995;20: 635-9.

10. Guo HR, Tanaka S, Halperin WE, Cameron LL. Back pain prevalence in US industry and estimates of lost workdays. Am J Public Health 1999;89:1029-35.

11. Manchikanti L. Role of neuraxial steroids in interventional pain management. Pain Physician 2002;5: 182-9.

12. Vroomen PC, de Krom MC, Slofstra PD, Knottnerus JA. Conservative treatment of sciatica: a systematic review. J Spinal Disord 2000;13:463-9.

13. Starkweather A, Witek-Janusek L, Matthews HL. Neural-immune interactions: implications for pain management in patients with low-back pain and sciatica. Biol Res Nurs 2005;6:196-206.

14. Pettersson K, Toolanen G. High-dose methylprednisolone prevents extensive sick leave after whiplash injury: a prospective, randomized, double-blind study. Spine 1998;23:984-9.

15. Haimovic IC, Beresford HR. Dexamethasone is not superior to placebo for treating lumbosacral radicular pain. Neurology 1986;36:1593-4.

16. Rydevik BL, Cohen DB, Kostuik JP. Spine epidural steroids for patients with lumbar spinal stenosis. Spine 1997;22:2313-7.

17. Ridley MG, Kingsley GH, Gibson T, Grahame R. Outpatient lumbar epidural corticosteroid injection in the management of sciatica. Br J Rheumatol 1988; 27:295-9.

18. Buchner M, Zeifang F, Brocai DR, Schiltenwolf M. Epidural corticorsteroid injection in the conservative management of sciatica. Clin Orthop Relat Res 2000;(375):149-56.

19. Carette S, Leclaire R, Marcoux S, et al. Epidural corticosteroid injections for sciatica due to herniated nucleus pulposus. N Engl J Med 1997;336:1634-40.

20. Watts RW, Silagy CA. A meta-analysis on the efficacy of epidural corticosteroids in the treatment of sciatica. Anaesth Intensive Care 1995;23:564-9.

21. Valet J-P, Giraudeau B, Rozenberg S, et al. Epidural corticosteroid injections for sciatica: a randomized, double blind, controlled clinical trial. Ann Rheum Dis 2003;62:639-43.

22. Radosevich DM, Husnick MJ. An abbreviated health status questionnaire: the HSQ-12. Update: The Newsletter of the Health Outcomes Institute 1995; 2:1-4.

23. Roland M, Morris R. A study of the natural history of back pain. Part 1: development of a reliable and sensitive measure of disability in low back pain. Spine 1983;8:141-4.

24. Fauci A, Braunwald E, Isselbacher K, et al, eds. Harrison's principles of internal medicine, 14th ed. New York (NY): McGraw-Hill and Companies, Inc.; 1998:2054-6.

25. Brown E, Suppes T, Khan D, Carmody T. Mood changes during prednisone bursts in outpatients with asthma. J Clin Psychopharmacol 2002;22:55-61.

26. Aaron S, Vandemheen K, Hebert P, et al. Outpatient oral prednisone after emergency treatment of chronic obstructive pulmonary disease. $\mathrm{N}$ Engl J Med 2003;348):2618-25.

27. Brown E, Beard L, Frol A, Rush A. Effect of two prednisone exposures on mood and declarative memory. Neurobiol Learn Mem 2006;86:28-34. 